\title{
Leukocyte marker CD43 promotes cell growth in co-operation with $\beta$-catenin in non-hematopoietic cancer cells
}

\author{
ANNA BALIKOVA ${ }^{1}$, KERSTI JÄÄGER ${ }^{2}$, JANELI VIIL ${ }^{1}$, TOIVO MAIMETS $^{1}$ and LILIAN KADAJA-SAAREPUU ${ }^{1}$ \\ ${ }^{1}$ Department of Cell Biology, Institute of Molecular and Cell Biology, University of Tartu, Riia 23, 51010 Tartu; \\ ${ }^{2}$ Institute of Gene Technology, Tallinn University of Technology, Akadeemia tee 15, 12618 Tallinn, Estonia
}

Received January 3, 2012; Accepted March 19, 2012

DOI: 10.3892/ijo.2012.1440

\begin{abstract}
The Wnt/ $\beta$-catenin pathway regulates key cellular processes such as differentiation, proliferation, apoptosis; and its activation promotes development of several cancer types. Expression of CD43 (leukosialin), the predominant leukocyte transmembrane sialoglycoprotein, has been detected in many tumors of non-hematopoietic origin. CD43 participates in cell adhesion and regulates intracellular signal transduction pathways involved in cell proliferation and survival. The cytoplasmic domain of CD43 has been reported to translocate to the nucleus, interact with $\beta$-catenin and affect its target gene expression, but the impact of this action on cell fate is still unknown. We demonstrate, here, by colony formation assay and siRNA-mediated gene silencing that CD43 and $\beta$-catenin co-operate in promoting cell growth. Moreover, in cells with down-regulated $\beta$-catenin expression the activation of $\mathrm{p} 53$ in response to CD43 overexpression is significantly impaired. In addition, the presence of both CD43 and $\beta$-catenin is required for the TCF/LEF-mediated transcription. Presumably, the fulllength CD43 participates in this transcriptional regulation. We show that the mature CD43 localizes to the nucleus, where it binds chromatin, co-localizes and co-immunoprecipitates with $\beta$-catenin, and enhances the reporter gene expression regulated by $\beta$-catenin. These observations provide clear evidence linking CD43 to the Wnt/APC/ $\beta$-catenin signaling pathway and supporting our hypothesis according to which CD43 plays a role in tumor development.
\end{abstract}

\section{Introduction}

CD43 (also known as leukosialin, sialophorin) is one of the most prevalent leukocyte transmembrane sialoglycoproteins expressed on cells of hematopoietic lineage including hema-

Correspondence to: Dr Anna Balikova, Department of Cell Biology, Institute of Molecular and Cell Biology, University of Tartu, Riia 23, 51010 Tartu, Estonia

E-mail: anna.balikova@gmail.com

Key words: leukosialin, sialophorin, CD43, $\beta$-catenin, cell growth, cell density, colon cancer topoietic stem cells (1) but excluding mature erythrocytes and B-cell subsets $(2,3)$. CD43 has a highly conserved cytoplasmic domain and mucin-like extracellular domain which is extensively $O$-glycosylated (4). The cytoplasmic domain of CD43 interacts with cytoskeletal proteins and regulates a variety of intracellular signal transduction pathways involved in cell activation, proliferation, and survival (5). Notably, the cross-linking of CD43 induces apoptosis of human hematopoietic progenitor cells but not stem cells (6). Whereas, in T-cells and monocytes the cross-linking of CD43 stimulates proliferation via protein kinase $\mathrm{C}(\mathrm{PKC})$ pathway (7). The high level of glycosylation and negative charge of the extracellular part of CD43 explain anti-adhesive properties of the CD43 molecule (8). CD43 also transmits signals enabling other ligand-receptor interactions to promote cell adhesion $(9,10)$ and may function as a ligand for ICAM-1 and E-selectin on endothelial cells $(11,12)$. Early expression of CD43 on hESC-derived hematopoietic progenitors may also indicate a possible role of CD43 in hematopoietic development, including acquisition of anti-adhesive properties by emerging hematopoietic cells (13).

Most of the work investigating CD43-mediated signaling has been done in the context of hematopoietic cells because CD43 expression has long been considered specific exclusively for immune cells. However, a number of studies demonstrate CD43 expression in different tumors of non-hematopoietic origin, including lung, breast and colon, but not in normal tissues (14-16). CD43 expression is also detected in several cancer cell lines (17-19). Aberrant expression and glycosylation of CD43 have been proposed to contribute to cancer progression $(15,20)$ and are associated with immune deficiency $(21,22)$. While in blood cells CD43 is expressed on the cell membrane, in nonhematopoietic cancer cells, in contrast, CD43 is located primarily intracellularly and even nuclearly (23). The cytoplasmic domain of CD43 contains a functional NLS (nuclear localization signal) and interacts with the nuclear transporter protein Ran which offers an explanation for the nuclear localization of CD43 (24). The translocation of the cytoplasmic part of CD43 to the nucleus is possible due to its $\gamma$-secretase-dependent proteolytic removal from the membrane. Human CD43 is processed by $\gamma$-secretase in several carcinoma cell lines (25). The resulting CD43 cytoplasmic tail has been shown to localize into a subnuclear structure, known as PML leukaemia nuclear body, and is involved in the regulation of apoptosis (26). Also, the cleavage in the extracellular domain of CD43 is well described $(27,28)$, and the 
soluble CD43 ectodomain fragment has been detected in human plasma (29). Therefore, CD43 might be involved in the regulated intramembrane proteolysis (RIP) signaling pathway similarly to Notch-1 (30), ErbB-4 (31), CD44 (32). In RIP signaling the intramembrane cleavage does not take place until the bulk of the protein on the extracytosolic (luminal or extracellular) face has been removed by a primary cleavage. This primary cleavage can occur in the lumen of the ER, in a post-ER compartment, or at the cell surface. The secondary intramembrane cleavage of type I transmembrane proteins, among which belongs CD43, requires presenilin-1 that cleaves off the cytoplasmic tail a few amino acids into the membrane. The released cytoplasmic fragment has in some cases been shown to translocate into the nucleus where it affects gene activation (33).

In our previous studies on the function of CD43 in nonhematopoietic cancer cells we have identified CD43 as a potential oncogene. Its overexpression causes accumulation of the tumor suppressor protein p53 and induction of apoptosis which is an ARF-dependent process (34). Recently, we have shown that CD43 increases the colony formation ability of both human and mouse cells due to increased growth rate but only in the absence of either p53 or ARF in the cells. Moreover, we found that cells overexpressing CD43 are more resistant to Fas-mediated apoptosis, the mechanism possibly giving these cells the growth advantage (35). In addition, CD43 has been suggested to have a role in Wnt $/ \beta$-catenin signaling as the cytoplasmic tail of CD43 interacts with $\beta$-catenin and promotes its target gene expression (24).

$\beta$-catenin, a potent proto-oncogene, is a multifunctional protein which is involved in embryonic development and renewal of adult tissue and which aberrant activation has been shown to play a critical role in the development of different cancers including colon $(36,37)$. $\beta$-catenin belongs to the cellcell adhesion apparatus, and the signaling pool of $\beta$-catenin is a key component of the complex network of proteins acting in the Wnt signaling pathway. Upon Wnt signaling $\beta$-catenin translocates to the nucleus where it interacts with T-cell factor (TCF) and lymphocyte-enhancing factor (LEF) family transcription factors and with other transcriptional co-factors to form complexes that regulate genes important for proliferation, differentiation, and apoptosis $(36,38)$. The functioning of $\beta$-catenin is controlled by a large number of binding partners that affect the stability and the localization of $\beta$-catenin. Thereby, $\beta$-catenin is able to participate both in cell adhesion and in gene expression processes.

In this study our aim was to investigate the involvement of CD43 in tumorigenesis focusing on the cross-talk between CD43- and $\beta$-catenin-mediated signaling. Based on published evidence by our group and others and our preliminary results, we hypothesize that CD43 promotes cell growth in co-operation with $\beta$-catenin and this in turn leads to the induction of ARF/ p53-dependent apoptosis which is the main mechanism against malignant growth.

We used colon carcinoma cell line HCT116 with an increased $\mathrm{Wnt} / \beta$-catenin activity, a non-small lung cancer cell line H1299 where Wnt/ $\beta$-catenin signaling is very low, and a colon adenocarcinoma cell line COLO205 which expresses endogenous CD43 at a high level (17). We used siRNA-mediated gene silencing to knock down $\beta$-catenin or CD43 expression and examine the role of either protein by different experimental methods. We also applied subcellular fractionation and subsequent immunoprecipitation of CD43 and $\beta$-catenin in order to provide more detailed information about the nature of CD43 and $\beta$-catenin interaction.

The data gathered so far support our hypothesis according to which CD43- and $\beta$-catenin-mediated pathways interact to regulate cell growth towards survival and/or proliferation. The interaction between CD43 and $\beta$-catenin links CD43 to $\mathrm{Wnt} / \mathrm{APC} / \beta$-catenin signaling pathway and provides additional evidence on the involvement of CD43 in tumor development.

\section{Materials and methods}

Cell culture and transfections. The H1299 (p53-null human nonsmall cell lung carcinoma), COLO205 (mutant p53 expressing colon adenocarcinoma) and HCT116 (human ARF-null and wild-type p53 expressing colon carcinoma) cell lines (originally obtained from the American Type Culture Collection) were cultured in $5 \% \mathrm{CO}_{2}$ at $37^{\circ} \mathrm{C}$ in Iscove's modified DMEM (PAA) supplemented with $10 \%$ fetal bovine serum and penicillin/ streptomycin (both $100 \mathrm{U} / \mathrm{ml}$ ) (PAA).

Plasmid transfections were performed using the electroporation method as described earlier (39) or with ExGen 500 in vitro Transfection Reagent according to the manufacturer's instructions (Fermentas). Human wild-type TP53 in pCG vector and human $S P N$ in pcDNA vector have been previously described $(34,39)$. Human $C T N N B 1$ in pCI-neo vector encoding mutated $\beta$-catenin (S33Y) was obtained from Dr B. Vogelstein. The pEGFP-F vector expressing farnesylated EGFP was purchased from Clontech. The sequences of the human MDM2 and CDKN1A promoters were cloned into the promoterless luciferase reporter plasmid pGL-3-basic (Promega). The TOPflash reporter plasmid containing three optimal TCF-binding motifs upstream of a minimal FOS promoter (40) and FOPflash plasmid containing mutant TCF-binding sites, both driving luciferase expression, were purchased from Upstate (Millipore). Renilla luciferase control reporter vector pRL-TK containing the herpes simplex virus-thymidine kinase (HSV-TK) promoter was purchased from Promega.

siRNA transfections were performed using HiPerFect Transfection Reagent according to the manufacturer's instructions (Qiagen). Cells were first transfected with siRNA and $24 \mathrm{~h}$ later with expression vectors. Cells were analyzed $48 \mathrm{~h}$ after siRNA treatment. siRNA oligomers targeting CD43 and $\beta$-catenin (Ambion) were as follows: 5'-GCAAACUCUCUAGG AUCCCtt-3' (sense) and5'-GGGAUCCUAGAGAGUUUGCtg-3' (anti-sense) for SPN (targeting second exon); 5'-GGUGGUGGU UAAUAAGGCUtt-3' (sense) and 5'-AGCCUUAUUAACCACC ACCtg-3' (anti-sense) for CTNNB1 (targeting fifth exon). A non-specific siRNA was used as a negative control (Ambion).

Colony formation assay. The colony formation ability of cells was estimated as described earlier (35).

Reporter assay. Luciferase reporter gene expression was determined with the dual-luciferase reporter assay system (Promega). The luciferase activity was normalized to Renilla luciferase activity from co-transfected internal control plasmid pRL-TK. 
Western blot analysis and antibodies. Western blot analysis was performed as previously described (34). The primary antibodies used were: $\mathrm{mAb}$ anti-CD43-4D2 (23), mAb anti- $\beta$-catenin (BD Biosciences), pAb anti-phospho p53Ser15 (Cell Signaling), mAb anti- $\beta$-actin (AC-15, Abcam), pAb anti-GRP78/BiP (Abcam), $\mathrm{mAb}$ anti-RNA polymerase II (4H8, Abcam) and pAb antihistone H3 (Abcam). The secondary antibodies used were: biotinylated goat anti-mouse (DakoCytomation), biotinylated goat anti-rabbit (DakoCytomation), horseradish peroxidase (HRP) conjugated with streptavidin (DakoCytomation) and HRP-conjugated goat anti-mouse (Pierce).

Fractionation and immunoprecipitation. Subcellular protein fractionation kit (Pierce) was used for preparing fractions from COLO205 cells. Protein concentration in fractions was estimated by BCA (bicinchoninic acid) protein assay (Pierce). Equal amounts of protein from each fraction were used for further applications. Immunoprecipitations were performed using sheep anti-mouse IgG coated Dynabeads M-280 (Dynal) [1 $\mu \mathrm{g}$ $\mathrm{mAb}$ anti-CD43-4D2, mAb anti-CD43-1G10 (BD Biosciences) or $\mathrm{mAb}$ to negative control for Mouse IgG1 (Abcam) per $10^{7}$ Dynabeads] or a co-immunoprecipitation kit (Pierce) $(20 \mu \mathrm{g}$ $\mathrm{mAb}$ anti-CD43-4D2 or mAb to negative control for Mouse IgG1 per $50 \mu$ l Agarose Resin slurry) following the manufacturer's instructions.

Immunofluorescence using confocal microscopy. Cells were grown on cover slides at high or low density, fixed with $4 \%$ paraformaldehyde for $30 \mathrm{~min}$ at room temperature and permeabilized with $0.2 \%$ Triton X-100 for $10 \mathrm{~min}$ on ice. Slides were blocked with normal goat serum for $1 \mathrm{~h}$ at room temperature. Target proteins were stained with anti- $\beta$-catenin pAb (Cell Signaling) and anti-CD43-4D2 mAb (23) or anti-CD43-L-10 $\mathrm{mAb}$ (Caltag) followed by incubation with the secondary antibodies Alexa Fluor 564-conjugated goat anti-mouse and Alexa Fluor 488-conjugated goat anti-rabbit (Molecular Probes). Slides were mounted with glycerol/1 M Tris $\mathrm{pH}$ 9.5 1:1 containing DAPI. Images were captured with a confocal laser scanning microscope (Olympus FluoView FV1000) using a 100x oil immersion objective and analyzed by Olympus FV1000 software FV10-ASW version 1.6a.

\section{Results}

CD43 and $\beta$-catenin co-operate in promoting colony formation. Aberrant activation of $\beta$-catenin-dependent signaling plays an important role in colon cancer development. It has been shown that inhibition of $\beta$-catenin expression reduces growth of colon cancer cells (41). We have shown previously, that CD43 overexpression promotes cell growth and down-regulation of its expression results in reduced cell growth (35).

We exploited siRNA-mediated gene silencing to explore whether CD43 and $\beta$-catenin co-operate in promoting colony formation ability of the cells. Using human cell lines, H1299 and HCT116, we knocked down $\beta$-catenin expression in the cells overexpressing CD43 and contrariwise, inhibited CD43 expression in $\beta$-catenin overexpressing cells, and estimated the colony formation efficiency. Results are shown in Fig. 1. In the cells treated with control siRNA there were more colonies formed in $\beta$-catenin and CD43 overexpressing cells which

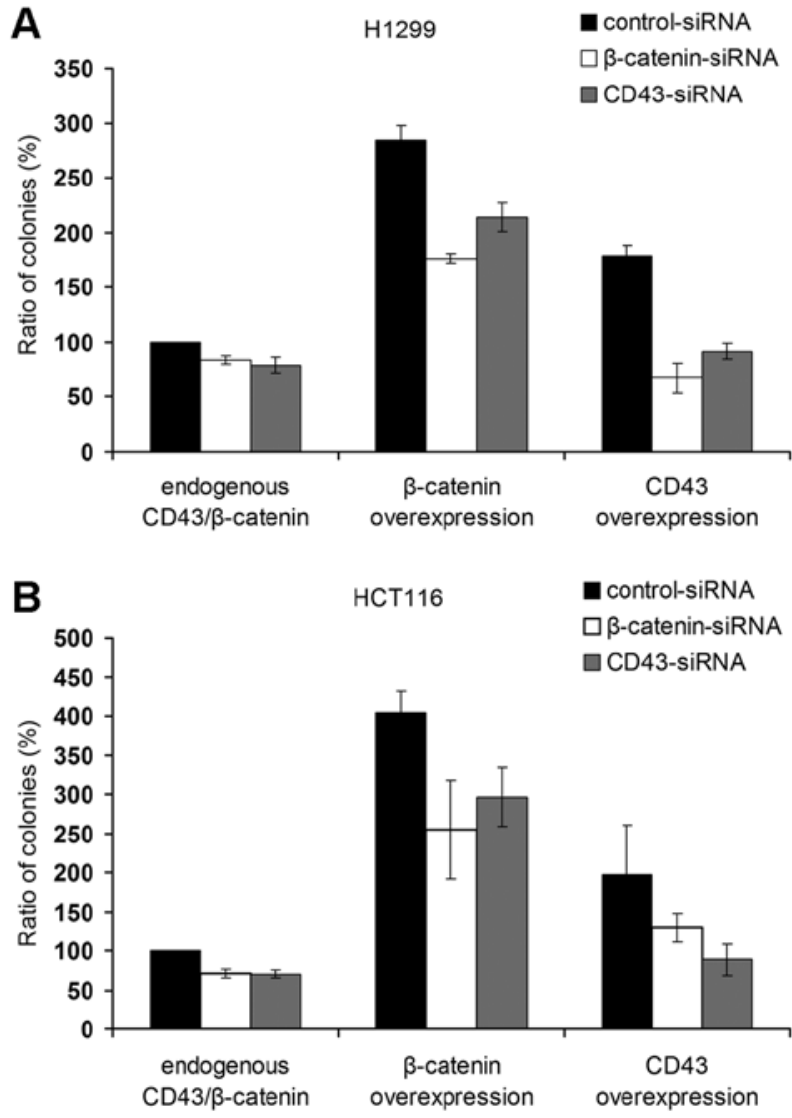

Figure 1. CD43 and $\beta$-catenin jointly promote colony formation. H1299 (A) and HCT116 (B) cells were transfected with indicated siRNAs followed by the transfection of plasmids expressing CD 43 or $\beta$-catenin. Data from three independent experiments are presented relative to the cells transfected with control siRNA.

is consistent with our previous results. Down-regulation of $\beta$-catenin expression resulted in the reduction of colony formation both in $\beta$-catenin and CD43 overexpressing cells. Also, the cells overexpressing CD43 or $\beta$-catenin formed fewer colonies when CD43 expression was inhibited.

These results suggest a role for CD43 in promoting cell growth in co-operation with $\beta$-catenin-mediated signaling because the cell growth-promoting property of CD43 overexpression was abolished by the down-regulation of $\beta$-catenin expression. Therefore, CD43 has a potential role in Wnt/ $\beta$-catenin signaling pathway, which is often de-regulated in human cancers.

CD43 requires $\beta$-catenin to induce p53-dependent response. We have previously shown that CD43 overexpression induces the accumulation of transcriptionally active p53, and this occurs due to the stimulatory effect of CD43 on cell growth $(34,35)$. As CD43 seems to co-operate with $\beta$-catenin in promoting colony formation, we tested whether it is also the case in p53 activation using luciferase reporter gene assay with p53-dependent MDM2 and CDKN1A promoters. The p53-null H1299 cells were treated with $\beta$-catenin siRNA or CD43 siRNA (as a control) followed by the co-transfection of expression plasmids and reporter constructs.

In the cells with decreased $\beta$-catenin expression the induction of the transcriptional activity of p53 in response to CD43 overexpression was inhibited (Fig. 2A and B). One can see that 

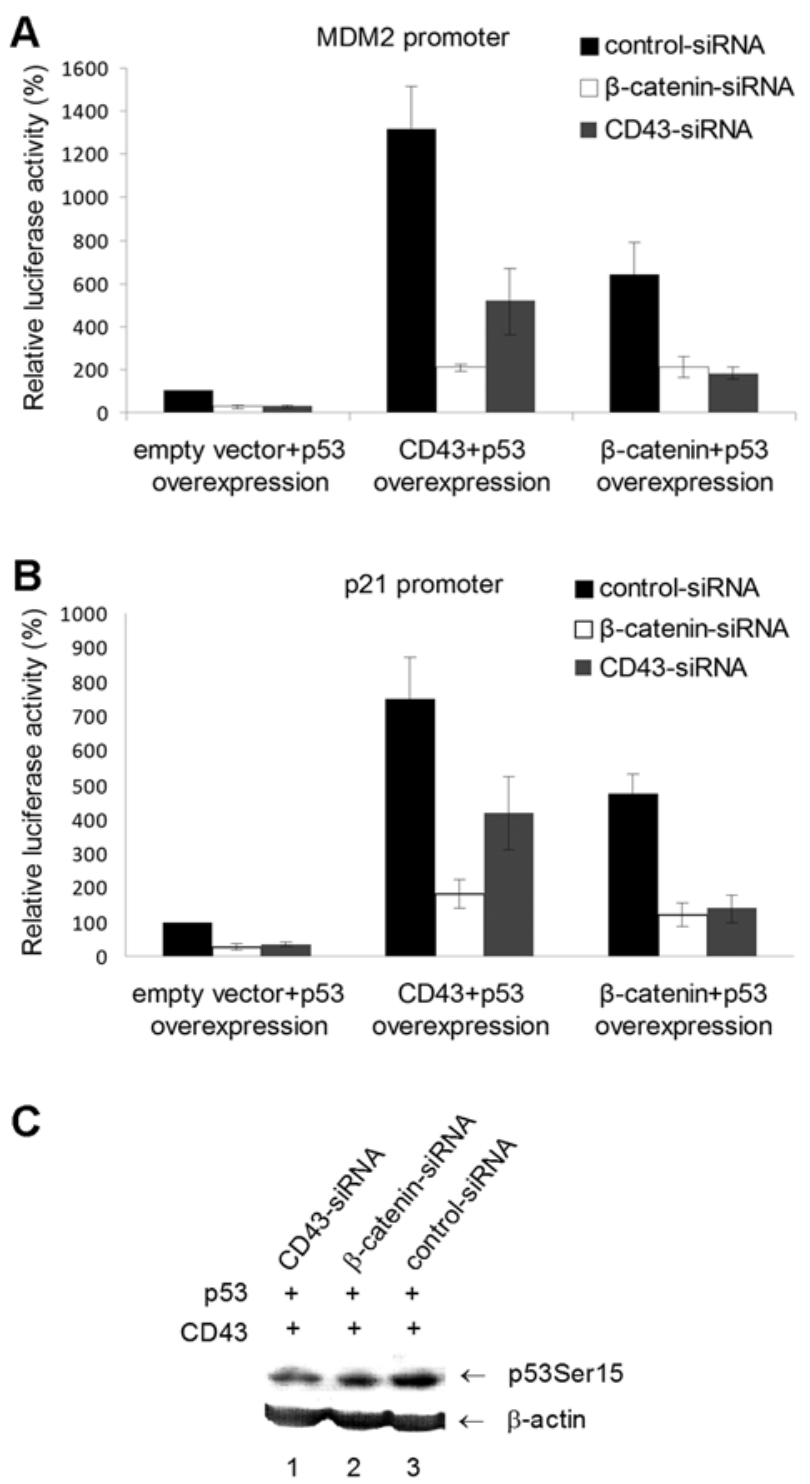

Figure 2. Activation of p53 in response to CD43 overexpression requires the presence of $\beta$-catenin. H1299 cells were transfected with indicated siRNAs followed by the transfection of p53 expressing plasmid together with plasmids expressing CD43 or $\beta$-catenin or empty vector control and with a reporter plasmid, containing either MDM2 (A) or CDKN1A (B) promoter. The results of three independent experiments are presented relative to the cells transfected with control siRNA, p53 expression plasmid, empty vector control and the reporter plasmid. (C), p53 protein expression levels and phosphorylation at serine 15 were analyzed by Western blot analysis in cells treated with different siRNAs and followed by co-expression of CD43 and p53. $\beta$-actin serves as an internal loading control.

even the basal activity of $\mathrm{p} 53$ is lower in the cells treated with siRNAs against $\beta$-catenin or CD43. The same cells were examined by Western blot analysis to determine $\mathrm{p} 53$ protein levels and phosphorylation at serine 15 . The latter indicates stabilization and activation of $\mathrm{p} 53$ probably due to the inhibition of MDM2-mediated degradation of p53 (42). Phosphorylation of serine 15 on $\mathrm{p} 53$ protein is induced in response to CD43 overexpression as well (34). Fig. 2C shows less phosphorylation at serine 15 when the expression of $\beta$-catenin or CD43 is down-regulated in the cells overexpressing CD43. Our results show that $\mathrm{CD} 43$ requires $\beta$-catenin to induce $\mathrm{p} 53$-dependent response.
Full-length CD43 enhances the reporter gene expression regulated by $\beta$-catenin. $\beta$-catenin is a transcriptional co-activator; it binds to the members of the TCF/LEF family of DNA-binding proteins forming a transcriptionally active complex (43). The activation of $\beta$-catenin/TCF/LEF-mediated transcription is the main down-stream consequence of Wnt signaling.

We studied the possible impact of CD43 on the transcriptional activity of $\beta$-catenin by a reporter assay in $\mathrm{H} 1299$ and HCT116 cells. We transfected cells with TOPflash luciferase reporter vector which reflects activation of LEF/TCF-sensitive transcription (40) together with EGFP expressing vector (negative control), $\beta$-catenin (positive control) or CD43 expressing vectors and measured luciferase activity. The overexpression of CD43 increased the transcription level of the reporter gene by several times compared to the control cells but not as much as the overexpression of $\beta$-catenin (Fig. 3A). The co-expression of both exogenous CD43 and $\beta$-catenin showed more pronounced luciferase activity relative to exogenous $\beta$-catenin alone (Fig. 3A).

To verify the role of CD43 in $\beta$-catenin-mediated transcriptional activation, we also assessed luciferase activity in case where either $\beta$-catenin or CD43 were down-regulated. The cells were treated with siRNA oligos followed by the transfection of expression vectors as described above. Indeed, the downregulation of CD43 expression resulted in lower luciferase activity both in H1299 and HCT116 cells (Fig. 3B and C). All reporter assays were repeated using FOPflash reporter vector, which contains mutant TCF-binding sites, as a negative control. Luciferase activities in the control assays were very low as expected (data not shown). These results demonstrate that in our experimental system the presence of both CD43 and $\beta$-catenin is required for the TCF/LEF-mediated transcription.

Full-length CD43 localizes to the nucleus, binds chromatin and interacts with $\beta$-catenin. Different CD43 molecules have been described in the cell: the mature full-length glycosylated CD43, the non-glycosylated CD43 precursor, the CD43-specific cytoplasmic tail fragment (denoted as the CD43-CTF), and the CD43 cytoplasmic tail (designated here as CD43ct). The CD43-CTF which is suggested to be formed by the proteolytic removal of the ectodomain includes a small part of the extracellular domain, the intact transmembrane and intracellular domains. The CD43ct is released as a result of the intramembrane $\gamma$-secretase cleavage that follows the cleavage in the extracellular domain (25).

Subcellular localization of a protein indicates its function to some extent. Therefore we investigated which CD43 molecules are present in different subcellular fractions. We used COLO205 cell line that expresses high levels of endogenous CD43. CD43 protein was visualized with the $\mathrm{mAb}$ anti-CD43-4D2 which reacts with all CD43 molecules of interest because the epitope is located near the C-terminal end of CD43 intracellular domain (aa 337-343). Surprisingly, the full-length glycosylated CD43 was detected in the soluble nuclear fraction and even at a more significant level in the chromatin-bound nuclear fraction (Fig. 4A). The precursor CD43 was found in the both nuclear fractions as well. The membrane fraction contained the highest amount of the precursor CD43 and the mature CD43. The precursor CD43 was absent from the soluble cytoplasmic frac- 

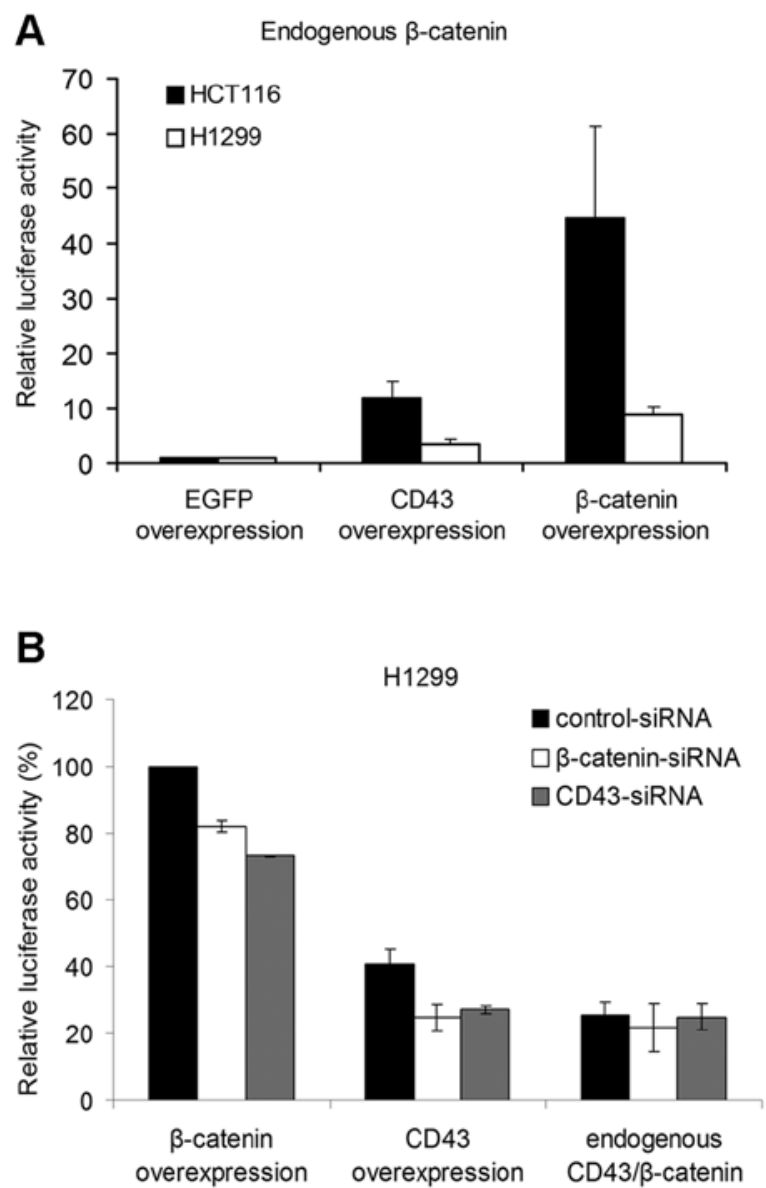

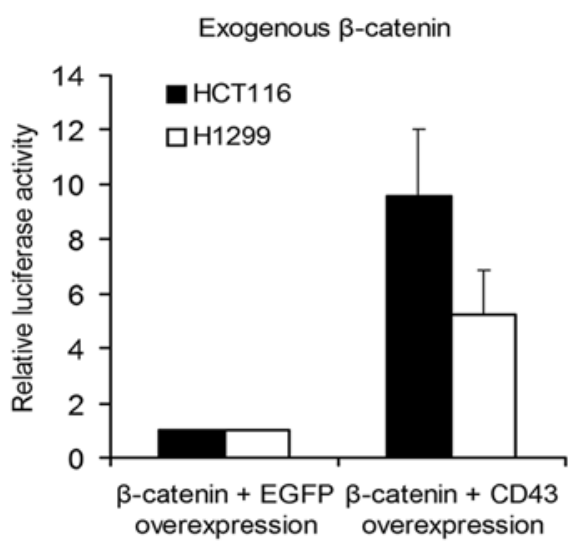

C

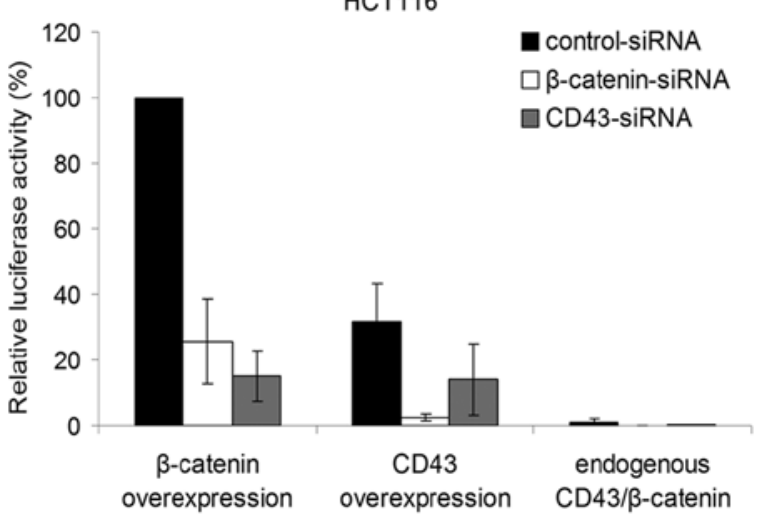

Figure 3. CD43 and $\beta$-catenin cooperate in the activation of TCF/LEF-dependent promoter. (A), H1299 and HCT116 cells were co-transfected with indicated expression vectors and TOPflash reporter plasmid. The results of three independent experiments are presented relative to the cells transfected with EGFP expression vector. (B), H1299 and (C), HCT116 cells were transfected with indicated siRNAs followed by the co-transfection of CD43 or $\beta$-catenin expression vectors and TOPflash reporter plasmid. The results of three independent experiments are presented relative to the cells transfected with control siRNA and $\beta$-catenin expression vector.
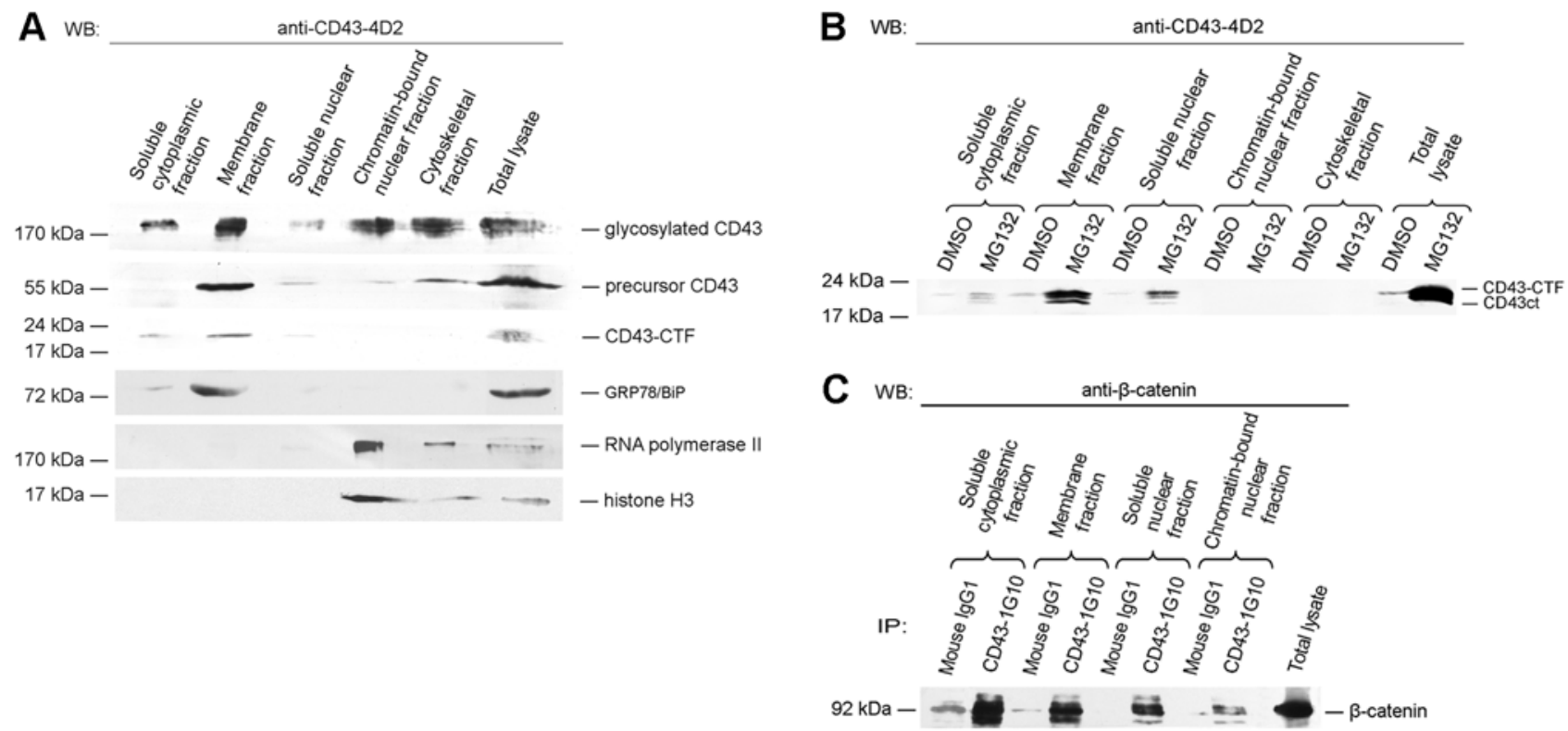

Figure 4. Full-length CD43 binds chromatin and interacts with $\beta$-catenin in the nucleus. (A), COLO205 cells were fractionated and examined by Western blot analysis using anti-CD43-4D2 mAb that recognizes all CD43 molecules in the cell. GRP78/BiP, a molecular chaperone of ER, RNA polymerase II and histone H3 were used to show fraction purity. (B), COLO205 cells were treated with the proteasome inhibitor MG132 $(20 \mu \mathrm{M})$ for $12 \mathrm{~h}$, fractionated and examined by Western blot analysis using anti-CD43-4D2 mAb. CD43 cytoplasmic fragments produced by intracellular cleavage are presented. DMSO was used as a negative control for MG132-treatment. (C), $\beta$-catenin co-immunoprecipitation with CD43 in subcellular fractions from COLO205 cells is shown by a precipitation with anti-CD43-1G10 $\mathrm{mAb}$ that recognizes the mature CD43 followed by a Western blot analysis probed with the $\mathrm{mAb}$ anti- $\beta$-catenin. The negative control for immunoprecipitation was Mouse IgG1 and the total lysate shows $\beta$-catenin migration. 

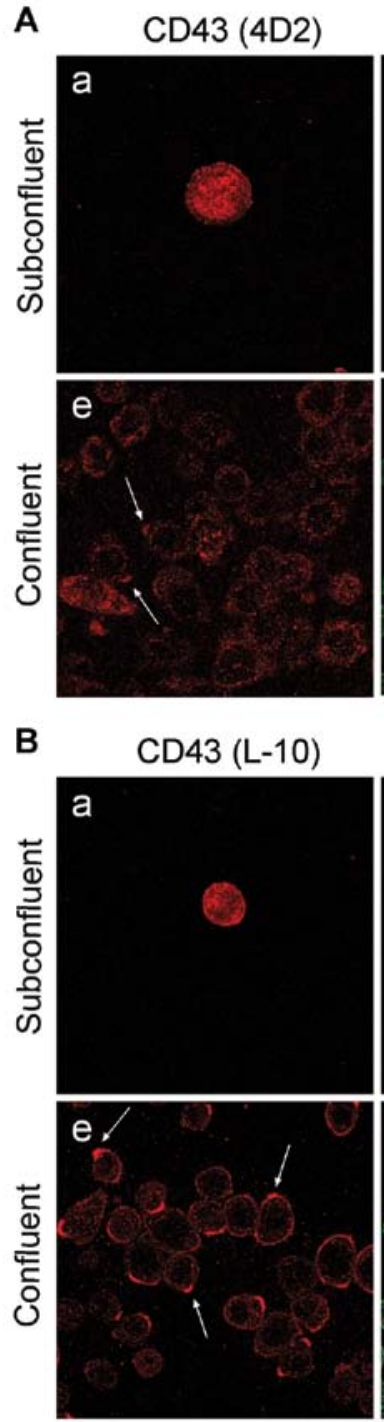

$\beta$-catenin
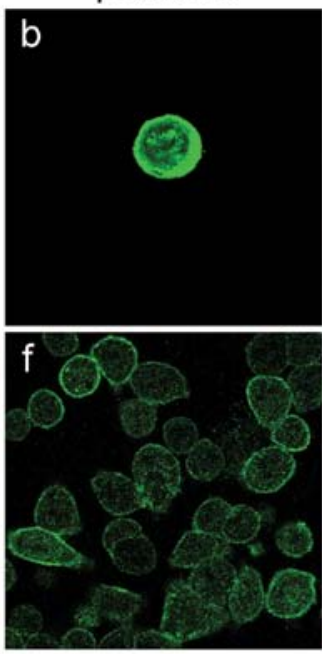

$\beta$-catenin
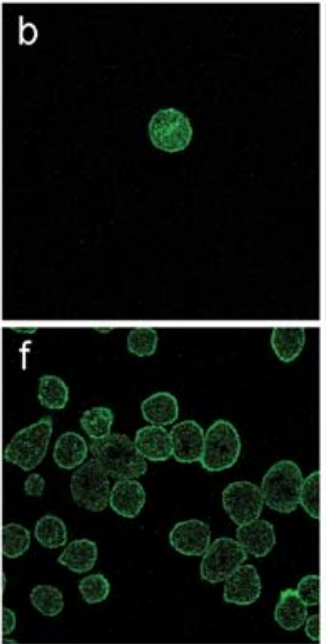

CD43 $+\beta$-catenin
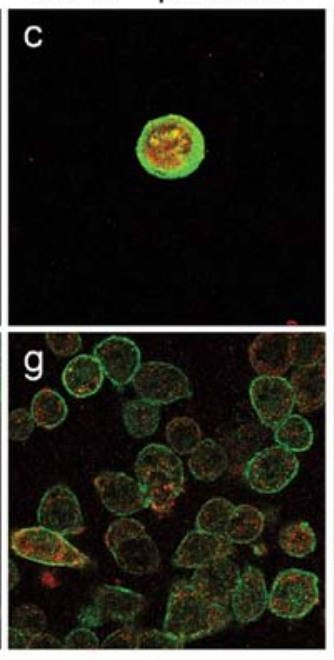

$\mathrm{CD} 43+\beta$-catenin
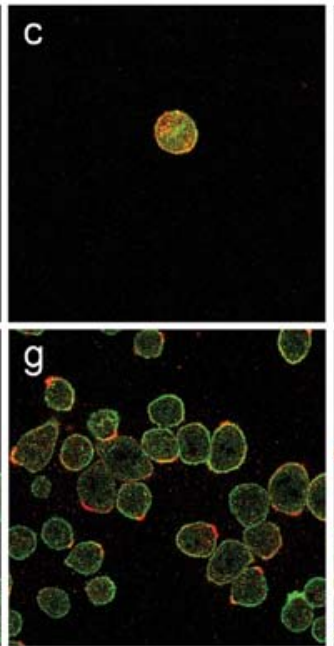

+ DAPI

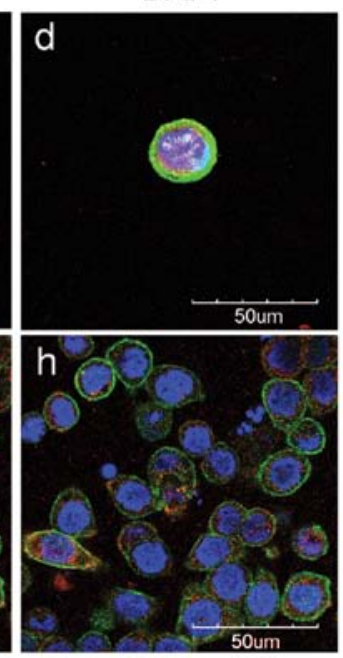

+ DAPI

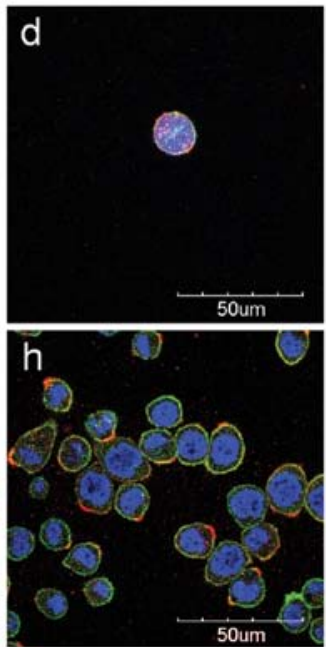

Figure 5. Confocal microscopy images of confluent and subconfluent cells showing partial co-localization between CD43 and $\beta$-catenin. (A), COLO205 cells at low density (a-d) and high density (e-h). The mAb anti-CD43-4D2 that recognizes all CD43 molecules in the cell was used to stain for CD43 (red). (B), COLO205 cells at low density (a-d) and high density (e-h). CD43 (red) was stained using the mAb anti-CD43-L-10 which recognizes only the full-length glycosylated CD43 protein. Green coloration represents $\beta$-catenin staining. 4',6-Diamidino-2-phenylindole (DAPI) was used to visualize nuclei (blue). The white arrows indicate CD43 focuses.

tion, which indicates that the precursor is compartmentalized in the cell apparently being incorporated into the endoplasmic reticulum (ER) and the Golgi apparatus for glycosylation. A considerable amount of the precursor and the mature CD43 proteins was detected in the cytoskeletal protein fraction. This is consistent with the previous findings showing that the cytoplasmic domain of CD43 associates with the cytoskeleton via ERM family adapter proteins (44). The smaller CD43 fragment, supposedly the CD43-CTF that has been shown to migrate at an apparent mass of approximately $20 \mathrm{kDa}$ (in COLO205 cell line slightly higher) (25), was observed in the soluble cytoplasmic fraction, in the membrane fraction and very poorly in the soluble nuclear fraction.

When COLO205 cells were incubated with the proteasome inhibitor MG132, the amount of the CD43-CTF increased as previously described (25), and even smaller CD43 fragments could be detected (Fig. 4B). We believe that the lower band corresponds to the cytoplasmic tail of CD43, because the expected difference in molecular weight between the CD43-CTF and the CD43ct is approximately $2.5 \mathrm{kDa}$ [calculated using Protein Molecular Weight Calculator at http://www.sciencegateway. org and potential cleavage sites described in (29) and (25)]. The CD43ct has been previously detected by immunoblotting only as a recombinant protein probably due to the rapid degradation of the native form (25). Upon addition of the proteasome inhibitor none of these CD43 molecules translocate to the chromatinbound nuclear fraction; the CD43 fragments appeared only in the fractions where CD43-CTF was present. These results indicate that the mature CD43 might have a role in regulating gene expression in the nucleus. This is consistent with our previous finding indicating that the cells overexpressing the full-length CD43, and not the CD43ct, are more resistant to Fas-mediated apoptosis (35).

In order to investigate whether the mature CD43 binds to $\beta$-catenin and in which part of a cell the interaction might take place, we performed a co-immunoprecipitation from the different subcellular fractions of COLO205 cells. CD43 was immunoprecipitated using the mAb anti-CD43-1G10 which 
recognizes only the full-length glycosylated CD43 protein. The immunoprecipitation was followed by a Western blot analysis using the $\mathrm{mAb}$ anti- $\beta$-catenin. Mouse IgG1 was used as a negative control. $\beta$-catenin was found to be co-immunoprecipitated with CD43 from all fractions isolated (Fig. 4C). The interaction between the mature CD43 and $\beta$-catenin in the chromatinbound nuclear fraction suggests that the full-length CD43 might modulate $\beta$-catenin/TCF/LEF-mediated transcription and contribute to impaired Wnt signaling in colon cancer.

Cell density-dependent interaction between CD43 and $\beta$-catenin. CD43 and $\beta$-catenin are both involved in cell adhesion. Moreover, cell density-dependent subcellular localization of CD43 (24) and $\beta$-catenin (45) has been reported before. We studied CD43 and $\beta$-catenin co-localization by confocal microscopy in confluent and subconfluent COLO205 cells. CD43 was detected with the $\mathrm{mAb}$ anti-CD43-4D2 that reacts with all CD43 molecules in the cell (Fig. 5A) or with the glycosylation specific $\mathrm{mAb}$ anti-CD43-L-10 that recognizes only the fulllength CD43 because its epitope is located in the extracellular part of CD43 (aa 1-78) (Fig. 5B). Our results demonstrate that CD43 and $\beta$-catenin partly co-localize and the co-localization pattern depends on cell density (Fig. 5A, panels c and g; Fig. 5B, panels $\mathrm{c}$ and $\mathrm{g}$ ). A considerable portion of CD43 staining was intracellular as previously described for CD43 in cancer cells (23). In subconfluent populations CD43 was found throughout the cells, significantly concentrating into the nucleus (Fig. 5A, panels a and d; Fig. 5B, panels a and d). In confluent populations CD43 formed focuses (Fig. 5A, panel e; Fig. 5B, panel e). Previous reports also show granular localization of CD43 in cancer cells (23). Similarly to CD43 $\beta$-catenin was detected in the nucleus in subconfluent cells (Fig. 5A, panel b; Fig. 5B, panel b), whereas in confluent cells nuclear $\beta$-catenin staining decreased (Fig. 5A, panels $\mathrm{f}$ and h; Fig. 5B, panels $\mathrm{f}$ and $\mathrm{h}$ ). CD43 and $\beta$-catenin co-localization in subconfluent cells was clearly seen in the nucleus as yellow areas (Fig. 5A, panels c and d; Fig. 5B, panels c and d). In confluent cells CD43 and $\beta$-catenin co-localized more in the cytoplasm and membrane (Fig. 5A, panel g; Fig. 5B, panel g). Remarkably, the mature CD43 protein accumulated in the nucleus and co-localized with $\beta$-catenin in subconfluent cell populations. This is consistent with our hypothesis that the full-length glycosylated CD43 co-operates with $\beta$-catenin in the promotion of a better survival of cancer cells.

To address whether the intensity of interaction between CD43 and $\beta$-catenin depends on cell density, we performed a co-immunoprecipitation in confluent and subconfluent COLO205 cells. CD43 was immunoprecipitated using the $\mathrm{mAb}$ anti-CD43-4D2 followed by a Western blot analysis using the $\mathrm{mAb}$ anti- $\beta$-catenin. Mouse IgG1 was used as a negative control (data not shown). In confluent cells the level of $\beta$-catenin co-precipitated with CD43 was slightly increased (Fig. 6A). To further investigate the impact of cell density on the interaction between CD43 and $\beta$-catenin, we repeated the co-immunoprecipitation in subcellular fractions from confluent and subconfluent COLO205 cells. CD43 and $\beta$-catenin were found to interact in all fractions regardless of cell density (as previously shown with anti-CD43-1G10 mAb in Fig. 4C). Interestingly, CD43 binding to $\beta$-catenin in the soluble nuclear fraction and in the chromatin-bound fraction

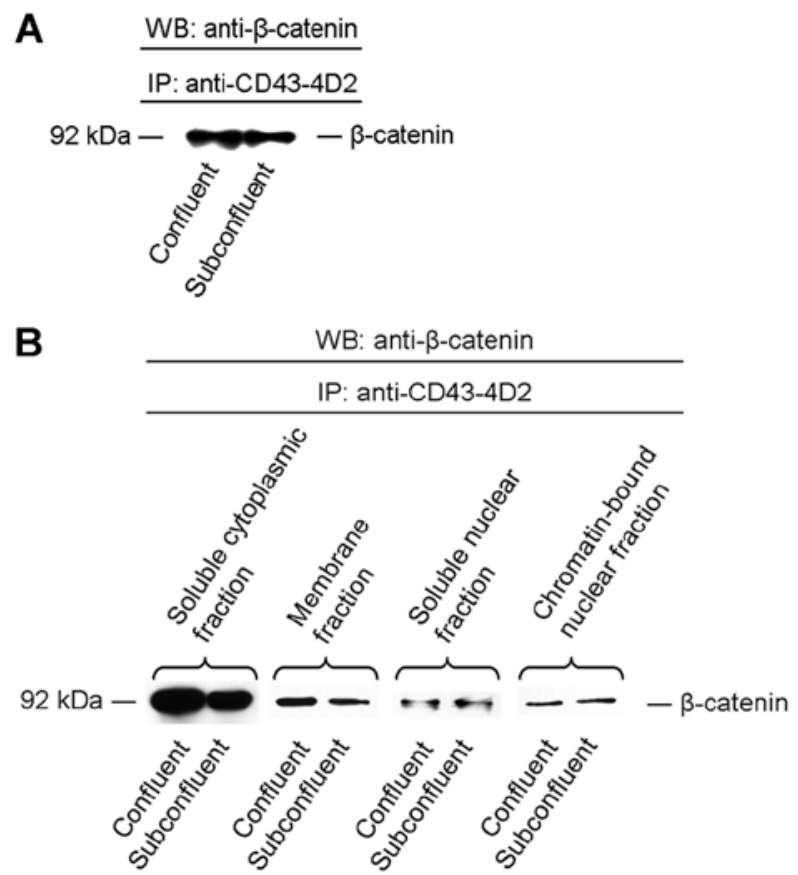

Figure 6. The influence of cell density on the interaction between CD43 and $\beta$-catenin. (A), CD43 and $\beta$-catenin co-precipitation from the total lysate of confluent and subconfluent COLO205 cells. (B), CD43 and $\beta$-catenin co-precipitation from the subcellular fractions of confluent and subconfluent COLO205 cells. CD43 was immunoprecipitated using the mAb anti-CD43-4D2 followed by a Western blot analysis using the anti- $\beta$-catenin $\mathrm{mAb}$. A negative control for a precipitation from each subcellular fraction was done using Mouse IgG1 (data not shown).

was equal in confluent and subconfluent cells (Fig. 6B). The difference between confluent and subconfluent cells appeared in the cytoplasmic fraction where CD43 binding to $\beta$-catenin was increased in confluent cells as compared to subconfluent cells (Fig. 6B). The discrepancy between co-localization and co-immunoprecipitation patterns might be conditioned by the fact that co-localization indicates only the potential to interact, which is verified here by co-IP.

$\beta$-catenin-mediated transcription depends on cell density. Colon cancer formation is commonly caused by the up-regulation of $\beta$-catenin/TCF/LEF-mediated transcription leading to the activation of genes that stimulate cell growth. Although we did not see any cell density-dependent changes in the interaction between CD43 and $\beta$-catenin in the nucleus, we studied the possible influence of cell density on $\beta$-catenin-mediated transcription. We transfected confluent and subconfluent COLO205, H1299 and HCT116 cells overexpressing CD43 with TOPflash luciferase reporter vector and measured luciferase activity. In COLO205 cell line there was a 2-fold higher transcription level of the reporter gene in subconfluent cells compared to confluent cells. In H1299 and HCT116 cells the effect was not so prominent (Fig. 7).

All reporter assays were repeated using FOPflash reporter vector as a negative control. Luciferase activities in the control assays were very low as expected (data not shown). These results indicate that in subconfluent cells transcriptionally active $\beta$-catenin accumulates in the nucleus. Thereby subconfluent cells might gain growth advantage over confluent cells. 


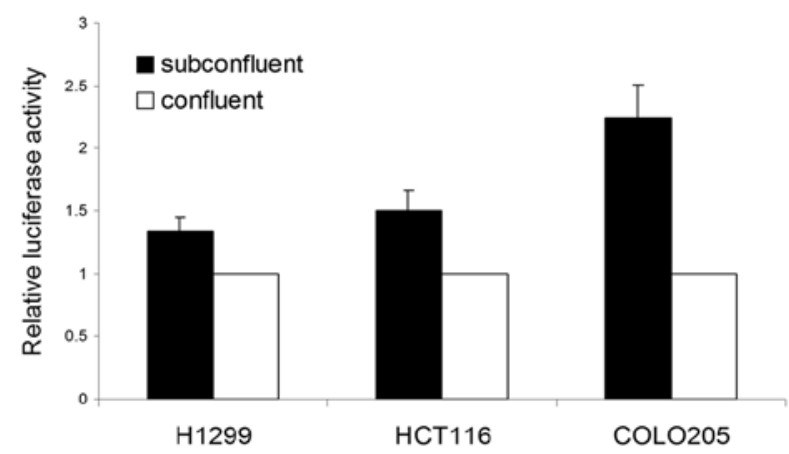

Figure 7. Transcriptional activity of $\beta$-catenin depends on cell density. TOPflash luciferase reporter vector was transfected into H1299, HCT116 or COLO205 confluent and subconfluent cells overexpressing CD43. The results of three independent experiments are presented relative to the confluent cells.

\section{Discussion}

The concept of deregulated activation of $\beta$-catenin as a principal cause of colorectal cancer is well accepted. Expression of the leukocyte marker CD43 in colon tumors but not in normal tissues has been described by different research groups. The contribution of CD43 to carcinogenesis is largely unknown, although some insights have been reported by our group and by others. Similarly to well-studied oncogenes (e.g., $\beta$-catenin) CD43 overexpression activates ARF/p53 tumorsuppressor pathway resulting in apoptosis. When either ARF or p53 is absent, CD43 overexpressing cells gain growth advantage due to the better survival of these cells. In addition, cytoplasmic tail of CD43 has been shown to interact with $\beta$-catenin and induce expression of its target genes (24). This suggests that CD43 is involved in colon tumor development via Wnt signaling pathway.

In the present study we provide new evidence for the interaction between CD43- and $\beta$-catenin-dependent signaling pathways. We address the possibility of the interaction between these two pathways by colony formation assay using siRNA against CD43 and $\beta$-catenin. We show that in both CD43- and $\beta$-catenin-overexpressing cells the depletion of either protein causes a reduction in the number of colonies. Besides, inhibition of $\beta$-catenin abrogates the growth-promoting effect of CD43 overexpression. The results confirm that both proteins are required for cell growth and CD43 is indeed involved in Wnt signaling.

Moreover, $\mathrm{CD} 43$ requires $\beta$-catenin to induce $\mathrm{p} 53$-dependent response. We have previously shown that the overexpression of the full-length CD43, but not the CD43ct-EGFP, causes the accumulation of $\mathrm{p} 53$ and apoptosis in the cells (34). $\beta$-catenin is able to activate p53 as well (46). Here we demonstrate that silencing $\beta$-catenin expression by siRNA inhibits the induction of the transcriptional activity of p53 in response to CD43 overexpression. Also, if expression of $\beta$-catenin or CD43 is down-regulated in the cells overexpressing CD 43 , the serine 15 on p53 protein is less phosphorylated, revealing the destabilization and deactivation of $\mathrm{p} 53$. This further confirms our hypothesis according to which CD43, as a potential oncogene, participates in signal transduction via Wnt-pathway.

The additional evidence supporting the role of CD43 in $\beta$-catenin-mediated signaling comes from our next finding employing the reporter assay. We show that the overexpression of $\mathrm{CD} 43$ enhances the reporter gene expression regulated by $\beta$-catenin. On the other hand, in the case where CD43 expression is silenced, the overexpression of $\beta$-catenin does not enhance the activation of its reporter gene expression, indicating important cross-talk between the CD43- and $\beta$-catenin-dependent pathways.

Applying fractionation and co-immunoprecipitation we have seen that the full-length glycosylated CD43 is able to localize into the nucleus where it binds chromatin and interacts with $\beta$-catenin. When cells were treated with the proteasome inhibitor MG132, two cytoplasmic fragments of CD43 became detectable in the soluble cytoplasmic fraction, in the membrane fraction and in the soluble nuclear fraction. We presume that the smaller fragment is the cytoplasmic tail of CD43, because it is an expected position for the CD43ct relative to the CD43-CTF $(25,29)$. Both of the fragments are produced from the cytoplasmic part of CD43 because the epitope of the anti-CD43-4D2 mAb used for detection is located in the distal C-terminus of CD43. In our experiments with MG132 proteasome inhibitor we have noticed that the intracellular domain of CD43 is cleaved in multiple sites (data not shown), which explains the appearance of the other fragment, but the biological meaning of this phenomenon is still unclear. The effect of the proteasome inhibitor implies that these CD43 molecules have a short protein half-life and are quickly degraded in the proteasome pathway. Besides, it has previously been proposed that CD43ct is quickly metabolized (25). Upon addition of the proteasome inhibitor none of these CD43 fragments translocate to the chromatin-bound nuclear fraction, the fragments appeared only in the fractions where CD43-CTF accumulated. This is somewhat contradictory to the published data indicating that $\mathrm{CD} 43 \mathrm{ct}$ binds $\beta$-catenin to activate the expression of $\beta$-catenin target genes $M Y C$ and cyclin D1 (24). However, the presence of the mature CD43 in the chromatinbound nuclear fraction and the failure of the CD43 cytoplasmic fragments to accumulate in this fraction correlate with our results showing that the overexpression of the full-length CD43, and not the CD43ct, helps the cells with defective ARF-p53 signaling to evade Fas-mediated apoptosis and promotes cell growth (35). $\beta$-catenin is also known to inhibit Fas expression on the cell surface (47). This supports the idea of the mature CD43 acting as a pro-survival factor via Wnt-pathway.

We propose that the CD43ct and the mature CD43 may have distinct functionality in the nucleus. The CD43ct, being SUMOylated and recruited into PML nuclear bodies (26), might participate in homeostasis and apoptosis primarily by interacting with PML-associated proteins (e.g., DAXX, p53, HIPK2) and the mature CD43, being capable of binding chromatin, regulates gene expression. However, the role of CD43 in $\beta$-catenin-mediated transcriptional activation is not fully understood. Still, we do not know whether CD43 acts as nothing but a chaperone/stabilizer for $\beta$-catenin and this way contributes to its transcriptional activity, or CD43 belongs to the protein complex that binds to the promoter regions of $\beta$-catenin target genes. It is tempting to speculate that the CD43ct stabilizes $\beta$-catenin and this way causes the activation of MYC and cyclin D1 expression while the mature CD43 binds DNA and interacts with TCF/LEF/ $\beta$-catenin complex at promoter regions of $\beta$-catenin target genes. Besides, Seo and Ziltener demonstrated that the CD43ct is recruited into 
PML by confocal microscopy. However, they detected the fulllength glycosylated CD43 by Western blot analysis using the same antibody. In addition, they observe that the mature CD43 may be SUMOylated as well. Hence, it cannot be excluded that only the mature CD43, or the both molecules localize in PML nuclear bodies.

The mechanism for the translocation of the full-length glycosylated CD43 into the nucleus can be based on the presence of the NLS in the cytoplasmic domain of the protein (24) or on the reversible attachment of $O$-linked $\mathrm{N}$-acetylglucosamine $(O-G l c N A c)$ which is known to play an important role in the modulation of the biological activity of intracellular proteins. The involvement of $O$-GlcNAc in the NLS-independent nuclear transport of cytosolic proteins was first sustained by studies on bovine serum albumin showing that the protein could be actively carried to the nucleus when it was modified with sugars (48). One of the mechanisms through which $O$-GlcNAc might act as a nuclear localisation signal is by counteracting the function of phosphorylation $(48,49)$. Phosphorylation has been shown to affect nuclear translocation of cytosolic proteins $(49,50)$. The localization of CD43 might be regulated by the same mechanism because CD43 is phosphorylated and extensively $O$-glycosylated carrying core $2 O$-glycan structures which contain GlcNAc (51).

Moreover, the $O$-glycan structures of CD43 might account for oncogenic properties of the protein. It has been reported that abnormal expression level of certain $O$-glycan structures as well as occurrence of truncated forms, precursors, or novel structures of $O$-glycans may affect ligand-receptor interactions (e.g., modulating the binding to alternative ligands) and thus interfere with regulation of signal transduction (52). Indeed, aberrant glycosylation of proteins, including CD43, may contribute to cancer progression by modifying cell signaling, adhesion, migration and survival $(20,53,54)$. Tumor-specific glycoforms of CD43 are expressed in different carcinomas, but not in normal tissues from the same patients (55). Also, altered expression of distinct glycoforms of CD43 has been associated with neoplastic transformation $(14,15,23)$. Aberrant signaling of these CD43 glycoforms may promote tumorigenesis by activating $\beta$-catenin, NF- $\mathrm{BB}, \mathrm{NFAT}$ and $\mathrm{AP}-1$, which are prosurvival transcription factors and promote a tumor phenotype when deregulated $(25,56)$. In addition, proteolytic processing of certain proteins demonstrates a requirement for $O$-glycans at specific sites in order to prevent proteolytic cleavage which eliminates biological activity or prevents continued residence/ activity of the intact protein at its designated subcellular location (51). Thus, abnormal glycosylation could modulate the proteolytic processing of CD43 and therefore interfere with CD43 functionality.

Another factor which is able to modulate CD43 and $\beta$-catenin localization and function is cell density. Cell densitydependent subcellular localization of $\beta$-catenin and CD43 has been described before $(24,45)$. Meanwhile, it is widely accepted that the involvement of $\beta$-catenin in cell adhesion or transcription is strictly associated with the subcellular localization of $\beta$-catenin. Here, we demonstrate that in subconfluent cells CD43 and $\beta$-catenin co-localize in the nucleus where, based on our hypothesis, CD43 could contribute to tumor formation in co-operation with $\beta$-catenin. We had supposed that the amount of CD43 bound to $\beta$-catenin increases in subconfluent cells, but it was not confirmed by the co-immunoprecipitation experiment. Moreover, the co-immunoprecipitation from different subcellular fractions revealed equal intensity of CD43 and $\beta$-catenin interaction in the nucleus of confluent and subconfluent cells. Apparently, the interaction outside the nucleus correlates with cell density (probably due to the involvement of both CD43 and $\beta$-catenin in cell adhesion), whereas the interaction in the nucleus presumably serves the purpose of transcriptional activation of $\beta$-catenin/TCF target genes which might participate in the inhibition of apoptosis. Hence, the interaction between CD43 and $\beta$-catenin in the chromatin-bound fraction and the co-operation in transactivation offer a possible explanation for the mechanism of the previously described phenomenon. Namely, CD43 overexpression increased the growth rate of cells making them more resistant to Fas-mediated apoptosis (35). In spite of the co-immunoprecipitation results, we found, employing the reporter assay, that the ability of $\beta$-catenin to activate reporter gene transcription is increased in subconfluent cells compared to confluent cells suggesting that the abnormal activation of $\beta$-catenin in cancer cells is not conditioned exclusively by CD43 or that CD43 might influence $\beta$-catenin-mediated transcription indirectly.

Furthermore, in colorectal carcinomas nuclear accumulation of $\beta$-catenin occurs in cells found at the invasive front and in disseminated cells at the metastatic site. The expression of cytoplasmic and membranous $\beta$-catenin is seen in the central areas of the metastases and primary tumors (57). We believe that the conditions in a subconfluent cell population mimic the conditions of the disseminated cells during metastasis. Interestingly, the tumor cells at the invasive front and the disseminated cells at the metastatic site have lost their proliferative activity (57). This suggests that the contribution of $\beta$-catenin to cancerogenesis is implemented by a better survival of the cells with nuclear $\beta$-catenin and not by an enhanced proliferation of these cells. The ability of $\beta$-catenin to interact with CD43 which conveys a survival advantage and helps to evade apoptotic responses $(26,35)$, also the co-operation between $\beta$-catenin and CD43 in enhancing cell growth is consistent with this hypothesis.

In conclusion, we have established a relation between CD43and $\beta$-catenin-mediated signaling pathways which supports the idea that CD43 could have a role in tumor development.

\section{Acknowledgements}

This work was partly supported by Estonian Science Foundation grants nos. 7545 and 7578, European Commission grant no. 205419 (ECOGENE) to Estonian Biocentre, and European Social Fund grant no. 1.2.0401.09-0073 to the University of Tartu (Graduate School of Biomedicine and Biotechnology). We thank Dr G. Hansson and Ch. Andersson for the CD43 expression vector and Dr B. Vogelstein for $\beta$-catenin (S33Y) expression vector.

\section{References}

1. Moore T, Huang S, Terstappen LW, Bennett M and Kumar V: Expression of CD43 on murine and human pluripotent hematopoietic stem cells. J Immunol 153: 4978-4987, 1994. 
2. Remold-O'Donnell E, Zimmerman C, Kenney D and Rosen FS: Expression on blood cells of sialophorin, the surface glycoprotein that is defective in Wiskott-Aldrich syndrome. Blood 70: 104-109, 1987.

3. Wiken M, Bjorck P, Axelsson B and Perlmann P: Induction of CD43 expression during activation and terminal differentiation of human B cells. Scand J Immunol 28: 457-464, 1988.

4. Shelley CS, Remold-O'Donnell E, Davis AE III, et al: Molecular characterization of sialophorin (CD43), the lymphocyte surface sialoglycoprotein defective in Wiskott-Aldrich syndrome. Proc Natl Acad Sci USA 86: 2819-2823, 1989.

5. Ostberg JR, Barth RK and Frelinger JG: The Roman god Janus: a paradigm for the function of CD43. Immunol Today 19: 546-550, 1998.

6. Bazil V, Brandt J, Chen S, et al: A monoclonal antibody recognizing CD43 (leukosialin) initiates apoptosis of human hematopoietic progenitor cells but not stem cells. Blood 87 1272-1281, 1996.

7. Silverman LB, Wong RC, Remold-O'Donnell E, et al: Mechanism of mononuclear cell activation by an anti-CD43 (sialophorin) agonistic antibody. J Immunol 142: 4194-4200, 1989.

8. Manjunath N, Correa M, Ardman M and Ardman B: Negative regulation of T-cell adhesion and activation by CD43. Nature 377: 535-538, 1995.

9. Kuijpers TW, Hoogerwerf M, Kuijpers KC, Schwartz BR and Harlan JM: Cross-linking of sialophorin (CD43) induces neutrophil aggregation in a CD18-dependent and a CD18-independent way. J Immunol 149: 998-1003, 1992.

10. Sanchez-Mateos P, Campanero MR, Del Pozo MA and SanchezMadrid F: Regulatory role of CD43 leukosialin on integrin-mediated T-cell adhesion to endothelial and extracellular matrix ligands and its polar redistribution to a cellular uropod. Blood 86: 2228-2239, 1995.

11. Rosenstein Y, Park JK, Hahn WC, Rosen FS, Bierer BE and Burakoff SJ: CD43, a molecule defective in Wiskott-Aldrich syndrome, binds ICAM-1. Nature 354: 233-235, 1991.

12. Matsumoto M, Atarashi K, Umemoto E, et al: CD43 functions as a ligand for E-selectin on activated T cells. J Immunol 175 : 8042-8050, 2005.

13. Vodyanik MA, Thomson JA and Slukvin II: Leukosialin (CD43) defines hematopoietic progenitors in human embryonic stem cell differentiation cultures. Blood 108: 2095-2105, 2006.

14. Santamaria M, Lopez-Beltran A, Toro M, Pena J and Molina IJ: Specific monoclonal antibodies against leukocyte-restricted cell surface molecule CD43 react with non hematopoietic tumor cells. Cancer Res 56: 3526-3529, 1996.

15. Sikut R, Nilsson O, Baeckstrom D and Hansson GC: Colon adenoma and cancer cells aberrantly express the leukocyte-associated sialoglycoprotein CD43. Biochem Biophys Res Commun 238: 612-616, 1997.

16. Pimenidou A, Madden LA, Topping KP, Smith KA, Monson JR and Greenman J: Novel CD43 specific phage antibodies react with early stage colorectal tumours. Oncol Rep 11: 327-331, 2004.

17. Baeckstrom D, Zhang K, Asker N, Ruetschi U, Ek M and Hansson GC: Expression of the leukocyte-associated sialoglycoprotein CD43 by a colon carcinoma cell line. J Biol Chem 270 : 13688-13692, 1995

18. Fernandez-Rodriguez J, Andersson CX, Laos S, et al: The leukocyte antigen CD43 is expressed in different cell lines of non hematopoietic origin. Tumour Biol 23: 193-201, 2002.

19. Ziprin P, Alkhamesi NA, Ridgway PF, Peck DH and Darzi AW: Tumour-expressed CD43 (sialophorin) mediates tumour mesothelial cell adhesion. Biol Chem 385: 755-761, 2004.

20. Fernandez-Rodriguez J, Dwir O, Alon R and Hansson GC: Tumor cell MUC1 and CD43 are glycosylated differently with sialyl-Lewis a and $\mathrm{x}$ epitopes and show variable interactions with E-selectin under physiological flow conditions. Glycoconj J 18: 925-930, 2001

21. Lefebvre JC, Giordanengo V, Limouse M, et al: Altered glycosylation of leukosialin, CD43, in HIV-1-infected cells of the CEM line. J Exp Med 180: 1609-1617, 1994

22. Khan S, Holding S, Dore PC and Sewell WA: Abnormal O-glycosylation of CD43 may account for some features of Wiskott-Aldrich syndrome. Med Hypotheses 70: 269-272, 2008.

23. Sikut R, Andersson CX, Sikut A, Fernandez-Rodriguez J, Karlsson NG and Hansson GC: Detection of CD43 (leukosialin) in colon adenoma and adenocarcinoma by novel monoclonal antibodies against its intracellular domain. Int J Cancer 82: $52-58,1999$.
24. Andersson CX, Fernandez-Rodriguez J, Laos S, et al: CD43 has a functional NLS, interacts with beta-catenin, and affects gene expression. Biochem Biophys Res Commun 316: 12-17, 2004.

25. Andersson CX, Fernandez-Rodriguez J, Laos S, Baeckstrom D, Haass C and Hansson GC: Shedding and gamma-secretasemediated intramembrane proteolysis of the mucin-type molecule CD43. Biochem J 387: 377-384, 2005.

26. Seo W and Ziltener HJ: CD43 processing and nuclear translocation of CD43 cytoplasmic tail are required for cell homeostasis. Blood 114: 3567-3577, 2009.

27. Bazil V and Strominger JL: Metalloprotease and serine protease are involved in cleavage of CD 43, CD44, and CD16 from stimulated human granulocytes. Induction of cleavage of L-selectin via CD16. J Immunol 152: 1314-1322, 1994.

28. Baeckstrom D: Post-translational fate of a mucin-like leukocyte sialoglycoprotein (CD43) aberrantly expressed in a colon carcinoma cell line. J Biol Chem 272: 11503-11509, 1997.

29. Schmid K, Hediger MA, Brossmer R, et al: Amino acid sequence of human plasma galactoglycoprotein: identity with the extracellular region of CD43 (sialophorin). Proc Natl Acad Sci USA 89: 663-667, 1992.

30. Schroeter EH, Kisslinger JA and Kopan R: Notch-1 signalling requires ligand-induced proteolytic release of intracellular domain. Nature 393: 382-386, 1998.

31. Ni CY, Yuan H and Carpenter G: Role of the ErbB-4 carboxyl terminus in gamma-secretase cleavage. J Biol Chem 278: 4561-4565, 2003.

32. Murakami D, Okamoto I, Nagano O, et al: Presenilin-dependent gamma-secretase activity mediates the intramembranous cleavage of CD44. Oncogene 22: 1511-1516, 2003.

33. Brown MS, Ye J, Rawson RB and Goldstein JL: Regulated intramembrane proteolysis: a control mechanism conserved from bacteria to humans. Cell 100: 391-398, 2000.

34. Kadaja L, Laos S and Maimets T: Overexpression of leukocyte marker CD43 causes activation of the tumor suppressor proteins p53 and ARF. Oncogene 23: 2523-2530, 2004.

35. Kadaja-Saarepuu L, Laos S, Jaager K, et al: CD43 promotes cell growth and helps to evade FAS-mediated apoptosis in nonhematopoietic cancer cells lacking the tumor suppressors p53 or ARF. Oncogene 27: 1705-1715, 2008

36. Tetsu $\mathrm{O}$ and McCormick F: Beta-catenin regulates expression of cyclin D1 in colon carcinoma cells. Nature 398: 422-426, 1999.

37. Giles RH, van Es JH and Clevers H: Caught up in a Wnt storm: Wnt signaling in cancer. Biochim Biophys Acta 1653: 1-24, 2003.

38. He TC, Sparks AB, Rago C, et al: Identification of c-MYC as a target of the APC pathway. Science 281: 1509-1512, 1998

39. Joers A, Kristjuhan A, Kadaja L and Maimets T: Tumour associated mutants of p53 can inhibit transcriptional activity of p53 without heterooligomerization. Oncogene 17: 2351-2358, 1998.

40. Korinek V, Barker N, Morin PJ, et al: Constitutive transcriptional activation by a beta-catenin-Tcf complex in APC-/- colon carcinoma. Science 275: 1784-1787, 1997.

41. Verma UN, Surabhi RM, Schmaltieg A, Becerra C and Gaynor RB Small interfering RNAs directed against beta-catenin inhibit the in vitro and in vivo growth of colon cancer cells. Clin Cancer Res 9: 1291-1300, 2003.

42. Giaccia AJ and Kastan MB: The complexity of p53 modulation: emerging patterns from divergent signals. Genes Dev 12 2973-2983, 1998

43. Barker N, Morin PJ and Clevers H: The Yin-Yang of TCF/betacatenin signaling. Adv Cancer Res 77: 1-24, 2000.

44. Yonemura S, Hirao M, Doi Y, et al: Ezrin/radixin/moesin (ERM) proteins bind to a positively charged amino acid cluster in the juxtamembrane cytoplasmic domain of CD44, CD43, and ICAM-2. J Cell Biol 140: 885-895, 1998.

45. Dietrich C, Scherwat J, Faust D and Oesch F: Subcellular localization of beta-catenin is regulated by cell density. Biochem Biophys Res Commun 292: 195-199, 2002.

46. Damalas A, Ben-Ze'ev A, Simcha I, et al: Excess beta-catenin promotes accumulation of transcriptionally active p53. EMBO J 18: 3054-3063, 1999.

47. Deng J, Miller SA, Wang HY, et al: beta-catenin interacts with and inhibits NF-kappa B in human colon and breast cancer. Cancer Cell 2: 323-334, 2002.

48. Duverger E, Roche AC and Monsigny M: N-acetylglucosaminedependent nuclear import of neoglycoproteins. Glycobiology 6 : 381-386, 1996. 
49. Guinez C, Morelle W, Michalski JC and Lefebvre T: O-GlcNAc glycosylation: a signal for the nuclear transport of cytosolic proteins? Int J Biochem Cell Biol 37: 765-774, 2005.

50. Nardozzi JD, Lott K and Cingolani G: Phosphorylation meets nuclear import: a review. Cell Commun Signal 8: 32, 2010.

51. Patsos $\mathrm{G}$ and Corfield A: O-glycosylation: structural diversity and functions. In: The Sugar Code: Fundamentals of Glycosciences. Gabius HJ (ed.) Wiley-VCH, Weinheim, pp111-138, 2009.

52. Ohtsubo K and Marth JD: Glycosylation in cellular mechanisms of health and disease. Cell 126: 855-867, 2006.

53. Hakomori S: Glycosylation defining cancer malignancy: new wine in an old bottle. Proc Natl Acad Sci USA 99: 10231-10233, 2002.

54. Brockhausen I: Mucin-type O-glycans in human colon and breast cancer: glycodynamics and functions. EMBO Rep 7: 599-604, 2006.
55. De Laurentiis A, Gaspari M, Palmieri C, et al: Mass spectrometry-based identification of the tumor antigen UN1 as the transmembrane CD43 sialoglycoprotein. Mol Cell Proteomics 10: M111.007898, 2011.

56. Santana MA, Pedraza-Alva G, Olivares-Zavaleta N, et al: CD43mediated signals induce DNA binding activity of AP-1, NF-AT, and NFkappa B transcription factors in human T lymphocytes. J Biol Chem 275: 31460-31468, 2000.

57. Brabletz T, Jung A, Reu S, et al: Variable beta-catenin expression in colorectal cancers indicates tumor progression driven by the tumor environment. Proc Natl Acad Sci USA 98: 10356-10361, 2001. 